\title{
STATISTICAL MODELS IN BIOMETRICAL GENETICS
}

\author{
J. A. NELDER
}

National Vegetable Research Station, Wellesbourne, Warwick

Received I.X.52

\section{INTRODUCTION}

THE statistical models belonging to the analysis of discontinuous variation in genetics have an elegance and exactness which are rare in the field of applied statistics. This is partly due to the qualitative nature of the characters usually investigated and partly to the random processes inherent in meiosis and fertilisation; both these properties of the experimental material make it possible to specify the hypothesis to be tested with great accuracy. The contrast between this state of affairs and that found when continuous variation obtains is indeed striking. The assumption that continuous variation represents the combined effect of polygenic inheritance and a blurring environmental overlay, though necessary as a basic postulate, is too vague to allow analysis to proceed very far. A further difficulty lies in the fact that experimental data on continuous variation, at least as obtained by present methods, do not suggest a detailed hypothetical structure which can be tested in future work. The hypotheses capable of being tested at present are relatively imprecise, and it must be expected, therefore, that the prediction value of analyses of continuous variation will be correspondingly less than that found in discontinuous variation. The analyses themselves will be less efficient, in the Fisherian sense, for the basic reason that the lack of plausible a priori structures for polygenic variation makes the construction of likelihood functions for sets of readings impossible, with the consequence that more or less inefficient ad hoc methods of analysis have to be used in default of anything better.

We shall consider in this paper the assumptions underlying the simpler models used in biometrical genetics and some of the statistical consequences of these models, with particular reference to the type of experiment in which the data relate to the descendants of a cross between two true-breeding lines. The discussion will be restricted to the analysis of first-order statistics (means) and second-order statistics (sums of squares and products) only.

\section{GENOTYPIC AND PHENOTYPIC EFFECTS}

The distinction between genotype and phenotype, that is, between genetic structure and its individual expression, is basic in genetics. In the analysis of continuously varying characters, it is usually said that we make the assumption that a measurement $(x)$ can be represented as the sum of a component due to genotype $(g)$ and a component 
due to environment $(e)$, i.e. $x=g+e$. It has been objected to this course in that the interaction between heredity cnd environment is so complex that its representation by a model such as $x=g+e$ is naive, even meaningless. It is worth while considering in more detail what meaning can be given to $g$ and $e$ in the above relation. It is clear that the idea of an ideal mean expression of a genotype is extremely vague. The combinations of environmental factors affecting development are practically infinite and there is no way in which we can weight the combinations in forming such a mean genotype. In any given experiment, however, the idea of randomisation can be used to define $g$. Consider an experiment in randomised blocks, in which each block contains an assemblage of plants of different genotypes, the numbers of each type being fixed beforehand. Under randomisation the plants of a given genotype in any block are distributed at random and give measurements $x_{1}, x_{2}, \ldots, x_{k}$, say, of some character. Writing $\bar{x}=\frac{\mathrm{l}}{k}\left(x_{1}+x_{2} \ldots+x_{k}\right)$ then $\tilde{x}$ is a random sample of one from the randomisation population of all possible arrangements of the $k$ plants in the block. Averaging over this population we may write $g=\mathrm{E}(\tilde{x})$; and $e=x_{i}-g$. Two consequences of this definition may be noted. First, $g$ as so defined is strictly local and must be expected to vary from experiment to experiment; secondly, we do not assume that the population of residuals is fixed by the experiment independently of the actual distribution of the genotypes in the block. The restrictiveness in the above definition of $g$ does not mean that no generalisation can be made from the experiment. For example, all variances and comparison of means are functions of differences of the $g$ 's and these may be expected to be more stable and so make possible a wider degree of generalisation. Even so, on general grounds we should expect that the differences of the $g$ 's will not be independent of the place and year of the experiment since the relative "fitness" of the different genotypes will be different under different external conditions.

The additional assumption that the residuals are independent of the distribution of the genotypes in a block greatly simplifies the analysis since this implies, among other things, that the " $e$ " or environmental component of variance is independent of the genetic or " $g$ " component, and hence in the expectation of all second-order statistics deriving from the same type of physical layout, the environmental component of variance is the same. This would still be true if the hypothetical populations of residuals of the various genotypes had the same variances without being necessarily identical, this being a slightly less restrictive assumption.

\section{Assumptions about the structure of $\mathbf{g}$}

Consider a character affected by $k$ loci. In general $g$ will be some function of the alleles at all the loci, i.e. we may write 
$g=g\left(\alpha_{1}, \alpha_{2}, \ldots, \alpha_{k}\right)$ where $\alpha_{i}, i=1,2, \ldots, k$, represents the state of locus $i$ in some genotype. The most obvious simplifying assumption here is that of independent action, which we may represent symbolically by $g\left(\alpha_{1}, \alpha_{2}, \ldots, \alpha_{k}\right)=g_{1}\left(\alpha_{1}\right)+g_{2}\left(\alpha_{2}\right)+\ldots+g_{k}\left(\alpha_{k}\right)$, so that the effect of each locus is independent of the state of the others.

Since such additivity is partly dependent on the scale of measurement, we are at liberty to adjust the scale to ensure at least average additivity, provided that the equal-variance property of the residuals over the genotypes is also preserved. Here we have a point of difference between the analysis of second-order statistics (as in biometrical genetics) and that of first-order statistics (as in, say, factorial manurial trials). In a factorial manurial trial, the absence of treatment interactions and the homogeneity of residual variances both simplify the analysis and interpretation but heterogeneity of residual variances does not, for example, bias the estimation of response slopes, although information may be lost. In biometrical genetics, additivity and the independence of $g$ of $e$ must both hold if the analysis is to proceed. This introduces a restriction on the characters which can be analysed with the present model ; how serious this restriction is remains to be seen.

For experiments on the generations following the crossing of two true-breeding strains, the following notation, developed by Fisher, Immer and Tedin (1932) and extended by Mather (1949) is convenient. For a gene pair $A-a$ (multiple allelomorphs are excluded by the derivations of all the plants from two true-breeding lines), the values of $g$ for $A A, A a$, aa, measured as a deviation from the mid-parent value are $d_{a}, h_{a},-d_{a}$ respectively. Given additivity, the value of $g$ for any genotype is the sum of the appropriate $-d$ 's, $h$ 's and $+d$ 's. The expectation of the genetic portion of any secendorder statistics can be expressed as a quadratic function of the $d$ 's and $h$ 's with coefficients depending on the recombination fractions and viability ratios of the different genes. When there is no differential viability, many (but not all) of the second-order statistics given by Mather are separable into two parts, one, denoted by $D$, depending only on the $d$ 's and the recombination fractions and the other, denoted by $H$, only on the $h$ 's and the recombination fractions.

\section{THE ESTIMATION OF $D, H$, AND $E$}

The experiments described by Mather consisted of blocks of plots, each plot containing an $F_{3}$ family, $F_{2}$ plants, $F_{1}$ or parent plants, etc. From each block second-order statistics are available, estimating the variance of $F_{2}$ plants, $F_{3}$ means, etc. The variance estimates include two measuring the non-heritable portion of the variance, one between plot means (taken from replicates of $F_{1}$ and parent plots within a block) and the other within plots (taken from the intra-plot variance of $F_{1}$ and parent plots). The expected values of the variance estimates are known linear functions of $D, H$ and $E$, and $D$ and $H$ may be 
heterogeneous due to linkage. There is a resemblance in the structure of this model to that used in "classical" least-squares analysis, where the expectations of certain observations are assumed to be known linear functions of some unknown parameters. There are, however, some differences too. In the least-squares analysis the "errors" in the observations (i.e. the differences from the expected values) have a variance matrix $V$ say, which is assumed known and in almost all practical cases it is assumed that $V=\sigma^{2} I$, where $I$ is the unit matrix. Here, the variance of the errors is constant and the observations are uncorrelated. The arithmetic in this case is reasonably tractable, but is much more tedious for a general $V$.

Deviations of $V$ from $\sigma^{2} I$ can be divided conveniently into two kinds, (i) the deviation of the leading diagonal terms (i.e. the variances) from equality, and (ii) the deviations of the other terms (i.e. the covariances) from zero. There are several reasons why it is likely that the variance matrix of the second-order statistics in biometrical genetical experiments will deviate from the unit matrix. Deviations of the first type would certainly occur under the normal model, where a variance estimate $x$ with expectation $\eta$ and based on $\nu$ d.f. has a variance $2 \eta^{2} / \nu$, which thus depends on both $\eta$ and $\nu$. Similar deviations are to be expected on the randomisation model. Deviations of the second kind will occur in the covariance of $V_{\overline{\mathrm{F}} 3}$ and $\bar{V}_{\mathrm{F} 3}$, and similar pairs. The correlation of the non-heritable components may be seen by considering $E_{1}$ and $E_{2}$. A uniform block will produce a small $E_{1}$ and $E_{2}$ and a variable block a large $E_{1}$ and $E_{2}$. We therefore expect a positive correlation between $E_{1}$ and $E_{2}$ as estimated from separate blocks. This is very marked in Mather and Philip's barley experiment (Mather, 1949) where the correlation is 0.9975 , which, although based only on five pairs of observations, is nevertheless significant. Similar positive correlations are to be expected between $V_{\mathrm{F} 2}$ and $E_{1}, V_{\overline{\mathrm{F}} 3}$ and $E_{2}$ for the same reason.

The effects of deviations from the assumed variance matrix in least-squares analysis are twofold, namely loss of efficiency in estimation and biasing of the standard errors. It should be noted that the estimates of $D, H$, etc. are not biased, but their sampling variances are inflated by the inefficient method of estimation. The bias in the standard errors is more serious because all significance tests are then unreliable.

The disturbing effects of the correlations mentioned above can be partly annulled by considering each block as a separate experiment with its own $E_{1}$ and $E_{2}$, these being now regarded as estimates of the randomisation variance within the block. In this way each block gives an estimate of $D$ and $H$ and standard errors can be obtained from the set of estimates by the usual methods. Until sufficient data are available to cast some light on the distribution of such estimates, this procedure must be regarded as a "large-sample" one.

Although this approach has the advantage that we avoid assump- 
tions about the variance matrix of the original statistics and assume only that the estimates of $D$ and $H$ from the various blocks can be regarded as samples from a normal population (or, at least, one for which the Central Limit Theorem holds), there are still difficulties remaining. Certain types of correlation are not eliminated, among them being that between $V_{\overline{\mathrm{F}} 3}$ and $\bar{V}_{\mathrm{F} 3}$ due to the heritable part of the variance and that between $E_{1}$ and $E_{2}$. Evaluation of the former type in special cases is tedious but in the case of one gene and with normal residuals the correlation does not appear to exceed 0.2 in absolute value. The correlation between $E_{1}$ and $E_{2}$ is for the most part a consequence of the lack of a randomisation set within plots. One way out of this difficulty would be a complete randomisation of all plants in a block so that, e.g. $\mathrm{F}_{1}$ plants would be found scattered over the block at random. Although such a complete randomisation might give rise to practical difficulties in some cases, it would have the analytical advantage in that there is now only one $E$ occurring in the analysis and its contributions to the statistics, though not equal, are of known relative weight. It should also be noted that randomisation generates correlations (usually negative but not necessarily so) between sum of squares having no measurements in common, such as, e.g. $V_{\mathrm{F} 2}$ and $V_{\overline{\mathrm{F}} 3}$. An extreme instance of this is provided by a variety trial in randomised blocks where no treatment differences exist. In this case the sum of the treatment and error sum of squares is a constant on the randomisation model so that their correlation is $-\mathrm{I}$. In general, however, such correlations will be insignificant provided that there are a large number of plants in a block and that the plants contributing to the two sums of squares form only a small proportion of the whole.

\section{Re-examination of Mather and Philip's Barley Experiment}

This experiment, fully recorded and analysed by Mather (I949) (see particularly pp. 97-IOI), used as the variable a discriminant function compounded of ear width, total ear length, and length of the central six internodes, derived from previous data. The experiment comprised five blocks and each block provided statistics $V_{\text {F2or }}$ $V_{\overline{\mathrm{F} 3}}, W_{\mathrm{F} 2 / \mathrm{F} 3}, \bar{V}_{\mathrm{F} 3}, E_{1}$ and $E_{2}$. The analysis made in Mather's book follows the usual least-squares procedure and regards the separate $E_{1}, E_{2}$ as samples from a population. We shall now consider an alternative analysis following the approach considered in the previous section. Although a least-squares analysis will be used for each block, it is not suggested that this will give the most accurate estimates of $D, H$, and $E$, and the inverse $c$-matrix (see, e.g. Fisher (I 948), pp. I $5^{6-}$ I64) will not be used to provide standard errors; which will be obtained from the sum of squares of the separate estimates from the five blocks. The estimates given by the method are unbiased as previously pointed out, and can be regarded as the best under the circumstances, bearing in mind that we have no prior information on the relative variances 
and covariances of the second order statistics. It should be said in advance that, qualitatively speaking, the conclusions drawn from the analysis in no way differ from those of Mather, and the re-analysis is made simply to demonstrate the method employed.

In making the analysis, opportunity has been taken to introduce a slight refinement in putting the expectation of $V_{\overline{\mathrm{F} 3}}$ equal to $\frac{1}{2} D+\frac{1}{16} H+\sigma^{\prime 2}+\frac{1}{n}\left(\frac{1}{6} D+\frac{1}{8} H+\sigma^{2}\right)$ where $n$ is the harmonic mean of the number of plants contributing to each mean, and $\sigma^{2}$ is defined as the variance component of plot means instead of the total variance of plot means. Thus (writing $\mathscr{E}$ for expectation)

$$
\begin{aligned}
& \mathscr{E}\left(E_{2}\right)=\sigma^{\prime 2}+\frac{\sigma^{2}}{n} \\
& \mathscr{E}\left(E_{1}\right)=\sigma^{2}
\end{aligned}
$$

$\sigma^{2}$ and $\sigma^{\prime 2}$ have been introduced for the two non-heritable components of variance in order that $E_{1}$ and $E_{2}$ may be reserved for the estimates and not used also for the population parameters. In order to avoid the recalculation of inverse matrices, $n$ has been taken as io throughout, though, owing to the loss of plants, this is not strictly true for each block.

Table I gives the values of the statistics for each block together

\begin{tabular}{|c|c|c|c|c|c|c|}
\hline \multirow{2}{*}{ Statistic } & \multicolumn{5}{|c|}{ Block } & \multirow{2}{*}{ Expectation } \\
\hline & A & B & C & D & E & \\
\hline $\begin{array}{l}V_{\mathrm{F} 2} \\
V_{\mathrm{F} 3} \\
W_{\mathrm{F} 2 / \mathrm{F3}} \\
\bar{V}_{\mathrm{F3}} \\
E_{1} \\
E_{2}\end{array}$ & $\begin{array}{r}9492 \cdot 0 \\
6289 \cdot 7 \\
6934 \cdot 6 \\
4513 \cdot 1 \\
1443 \cdot 8 \\
278 \cdot 6\end{array}$ & $\begin{array}{r}1540 \cdot 9 \\
5838 \cdot 6 \\
634^{2} \cdot 5 \\
4532 \cdot 5 \\
736 \cdot 9 \\
57 \cdot 9\end{array}$ & $\begin{array}{r}9179 \cdot 5 \\
5935 \cdot 1 \\
674^{2 \cdot 5} \\
4342 \cdot 4 \\
1433^{\circ} \cdot 1 \\
306 \cdot 8\end{array}$ & $\begin{array}{r}8673 \cdot 7 \\
7148 \cdot 8 \\
7439 \cdot 6 \\
4323 \cdot 1 \\
1720 \cdot 9 \\
403 \cdot 2\end{array}$ & $\begin{array}{r}9926 \cdot 4 \\
6013 \cdot 4 \\
6696 \cdot 8 \\
3867 \cdot 9 \\
747 \cdot 6 \\
54.9\end{array}$ & $\begin{array}{l}\frac{1}{2} D+1 H+\sigma^{2} \\
\frac{1}{2} D+\frac{1}{10} H+\sigma^{2}+\frac{1}{1}\left(G+\sigma^{2}\right) \\
\frac{1}{2} D+\frac{1}{8} H \\
G+\sigma^{2} \\
\sigma^{\prime 2}+r^{1} \sigma^{2} \\
\sigma^{2}\end{array}$ \\
\hline
\end{tabular}
with their expectations.

$G$ is the genetic portion of the variance within $\mathrm{F}_{3}$ families and $G=\frac{1}{4} D^{\prime}+\frac{1}{8} H^{\prime}$. In the absence of linkage $D^{\prime}=D, H^{\prime}=H$. In the presence of linkage, $D^{\prime} \neq D, H^{\prime} \neq H ; D^{\prime}$ and $H^{\prime}$ cannot be estimated separately in the absence of other statistics giving information about them.

Fitting one $D$, one $H$ and separate $E$ 's to the data and putting $G=\frac{1}{4} D+\frac{1}{8} H$, we find from least squares

$$
\begin{array}{llll}
\hat{D}=9602 \cdot 3, & \hat{H}=\mathrm{I} 5,270 \cdot 6, & E_{1 \mathrm{~A}}=840 \cdot 2 & E_{2 \mathrm{~A}}=106 \cdot 9 \\
E_{1 \mathrm{~B}}=1294 \cdot 0, & E_{2 \mathrm{~B}}=-274 \cdot 4, & E_{1 \mathrm{C}}=675 \cdot 6, & E_{2 \mathrm{C}}=-39 \cdot 9 \\
E_{1 \mathrm{D}}=596 \cdot 5, & E_{2 \mathrm{D}}=623 \cdot 1, & E_{1 \mathrm{E}}=537 \cdot 9, & E_{2 \mathrm{E}}=-112.9
\end{array}
$$

(where $\hat{D}$ and $\hat{H}$ denote the estimates of $D$ and $H$, and $E_{1 \mathrm{~A}}, E_{2 \mathrm{~A}}$ are the estimates of $E_{1}$ and $E_{2}$ in block A, etc.). 
The sum of squares of residuals is $10,526,166$. Fitting one $D$, one $H$, one $G$, and separate $E$ 's we find

$$
\begin{gathered}
\hat{D}=9800 \cdot 3, \quad \hat{H}=14,783 \cdot 0, \quad \hat{G}=3116 \cdot 0, \\
E_{1 \mathrm{~B}}=1699 \cdot 5, \quad E_{2 \mathrm{~B}}=-289 \cdot 6, \quad E_{1 \mathrm{C}}=1064 \cdot 4 \\
E_{1 \mathrm{D}}=\mathrm{I001} \cdot 9, \quad E_{2 \mathrm{D}}=608 \cdot 0, \quad E_{1 \mathrm{E}}=943 \cdot 3, \\
E_{1 \mathrm{~A}}=\mathrm{I} 245 \cdot 7, \quad E_{2 \mathrm{~A}}=9 \mathrm{I} \cdot 7 \\
E_{2 \mathrm{C}}=-53 \cdot 3, \\
E_{2 \mathrm{E}}=-\mathrm{I} 28 \cdot 0,
\end{gathered}
$$

with a sum of squares of residuals equal to $5,854,956$. The sum of squares due to fitting $G$, which measures the linkage effect, is the difference $10,526,166-5,854,956=4,67$ I, 2 10.

\section{Interaction of genetic components and blocks}

As defined in section 2, the genetic component $g$ of a measurement was strictly local in application and would be expected to vary with considerable changes in the environment. A test of the existence of such variation between blocks can be made by fitting separate $D$ 's and $H$ 's to each block and testing the further reduction in the sum of squares against the remainder. We also need the separate $D$ 's and $H$ 's for obtaining the standard errors. Such a test for interaction is not orthogonal to the linkage test but, as we shall see, there is little doubt as to the existence of linkage, and so a separate $D, H$ and $G$ will be fitted to each block. The results are given in table 2 .

TABLE 2

\begin{tabular}{|c|r|r|r|r|r|}
\hline \multirow{2}{*}{ Estimate } & \multicolumn{5}{|c|}{ Block } \\
\cline { 2 - 6 } & \multicolumn{1}{|c|}{$\mathrm{A}$} & \multicolumn{1}{|c|}{$\mathrm{B}$} & \multicolumn{1}{|c|}{$\mathrm{C}$} & \multicolumn{1}{|c|}{$\mathrm{D}$} & $\mathrm{E}$ \\
\hline & & & & \\
\hline$\hat{D}$ & & & & & \\
$\hat{H}$ & $10,516 \cdot 9$ & $5,915 \cdot 6$ & $9,968 \cdot 7$ & $13,852 \cdot 5$ & $8,748 \cdot \mathrm{I}$ \\
$\hat{G}$ & $11,678 \cdot 3$ & $30,390 \cdot 9$ & $11,744 \cdot 5$ & $1,029 \cdot 4$ & $19,072 \cdot 1$ \\
$\hat{E}_{1}$ & $3,112 \cdot 6$ & $3,712 \cdot 8$ & $2,967 \cdot 3$ & $2,679 \cdot 1$ & $3,108 \cdot 1$ \\
$\hat{E}_{2}$ & $1,386 \cdot 1$ & $847 \cdot 3$ & $1,355 \cdot 7$ & $1,618 \cdot 3$ & $763 \cdot 9$ \\
& $4 \cdot 3$ & $249 \cdot 3$ & $-22 \cdot 2$ & $-15 \cdot 1$ & $19 \cdot 3$ \\
\hline
\end{tabular}

The sum of squares of residuals is now $942,74^{2}$ giving $5,854,956-$ $94^{2,742}=4,912,214$ as the variation of $D, H, G$ within blocks. We therefore have the following analysis of variance

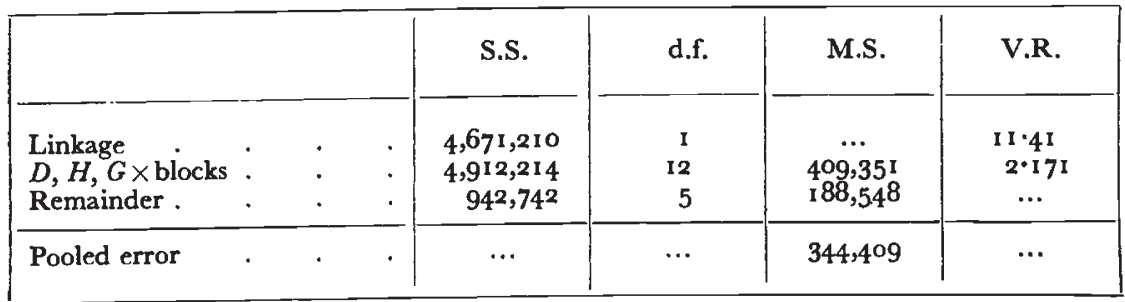


The interaction term is not significant and so we combine it with the remainder. Testing the linkage mean square against this combined remainder we obtain a variance ratio of $1 \mathrm{I} \cdot 4 \mathrm{I}$, for which $0.01>P>0.001$ so that there can be little doubt of the existence of linkage. For reasons previously given the significance level should be regarded as approximate only. Reverting to the values in table 2, the mean values of $D, H$ and $G$ for the five blocks are $\hat{D}=9800 \cdot 3$, $\hat{H}=14,783 \cdot 0, \hat{G}=3116 \cdot 0$ which are the values previously found from the fitting of a single $D, H$ and $G$, as indeed they must be, since each block carries equal weight in the experiment. From the sums. of squares and sums of products formed from table 2 we arrive at the following variance matrix for the estimates $\hat{D}, \hat{H}, \hat{G}$.

$$
\left[\begin{array}{rrr}
\mathrm{r}, 658,011 & -6,180,692 & -205,398 \\
& 23,501,304 & 787,612 \\
& & 28,462
\end{array}\right]
$$

From this we obtain, inter alia,

$$
\begin{aligned}
\text { s.e. } \hat{D} & = \pm 1287.6 \\
\text { s.e. } \hat{H} & = \pm 4847 \cdot 8 \\
\text { s.e. } \hat{G} & = \pm 168 \cdot 7 \\
\text { corr. }(\hat{D}, \hat{H}) & =-0.9901
\end{aligned}
$$

It will be noted that the standard errors for $\hat{D}$ and $\hat{H}$ are considerably higher than those obtained by Mather which were $808 \cdot 9$ and $2588 \cdot 4$ respectively. $\hat{H}$, however, remains significantly greater than zero.

\section{Interaction of genetic components with years}

As previously pointed out, an interaction between genotypic expression and years is to be expected on general grounds; such an interaction may not be detectable, however, if the years are too similar in their effects, or the extraneous variation swamps the year differences which do exist.

A possible result of such interaction would be that the $d$ 's and $h$ 's contributing to $W_{\mathrm{F} 2 / \mathrm{F} 3}$ are different from those contributing to, say, $V_{\text {F2 }}$. This can be tested by fitting a special constant for $W_{\mathrm{F} 2 / \mathrm{F} 3}$ and finding whether the sum of squares of residuals is thereby significantly reduced. This was done for the above data and gave a reduction in the sums of squares due to fitting $W$ as 38,6 r 8 , which was not significant. It was concluded, therefore, that no detectable interaction was present.

When several years' results are available, heterogeneity of $D$ and $H$ is best detected by comparing the results of fitting a single $D$ and $H$ to all the results and separate $D$ 's and $H$ 's for the separate years. (In the latter fitting, covariances such as $W_{\mathrm{F} 2 / \mathrm{F} 3}$ must have further separate constants fitted). 


\section{SUMMARY}

I. The statistical consequences of, and assumptions underlying, a frequently-used model in biometrical genetics, are considered.

2. Differences between this model and the "classical" leastsquares situation are discussed, and a new method of deriving estimates of the variance components and standard errors for these estimates is proposed.

3. A re-examination of Mather and Philip's barley data is made as an illustration of the method.

I wish to thank Professor K. Mather and Mr B. I. Hayman for reading the original draft of the paper, and for their valuable criticism and suggestions.

\section{REFERENCES}

FISHER, R. A. 1948. Statistical Mcthods for Research Workers. I oth ed. London. FISHER, R. A., IMMER, F. R., AND TEDIN, O. 1932. The genetical interpretation of statistics of the third degree in the study of quantitative inheritance. Genetics, $17,107-24$.

MATHER, к. 1949. Biometrical Genetics. London. 